\title{
Hipotireoidismo Neonatal Transitório Causado pelo Uso de Amiodarona Durante a Gestação - Relato de Dois Casos e Revisão da Literatura
}

\section{apresentação de caso}

\author{
Carla C. Pavan-Senn \\ SuzANa Nesi-França \\ Julita Pelaez \\ Rosana M. Pereira
}

Margaret C. DA S. BoguszeWSKI

ROMOlO SANDRINI Neto

LUIZ DE LACERDA FILHO

Unidade de Endocrinologia Pediátrica (UEP) do

Departamento de Pediatria do

Hospital de Clínicas da

Universidade Federal do Paraná, Curitiba, PR, Brasil.

Recebido em 28/03/2007 Aceito em 08/10/2007

\author{
RESUMO
}

Introdução: A amiodarona (AMD) é uma droga antiarrítmica que contém $37 \%$ de iodo. A AMD pode alcançar o feto por via transplacentária e causar hipotireoidismo congênito (HC) ou transitório (HCT). Relatamos dois casos de HCT em virtude de exposição gestacional à $A M D$, detectados pelo programa de triagem neonatal para HC no Estado do Paraná, Brasil. Caso clínico 1 (C1): TSH neonatal 78,2 mU/L (normal $<15 \mathrm{mU} / \mathrm{L}$ ). A AMD foi utilizada durante toda a gestação em virtude de arritmia materna. As dosagens séricas iniciais confirmaram o HC; e na primeira consulta [aos 14 dias de vida (dv)], foi iniciada levotiroxina (L-T $), 50 \mu \mathrm{g} / \mathrm{dia}$. Caso clínico 2 (C2): TSH neonatal $134 \mathrm{mU} / \mathrm{L}$. A AMD foi utilizada no último trimestre da gestação em virtude de arritmia materna. As dosagens séricas iniciais confirmaram o HC; aos $13 \mathrm{dv}$, foi iniciada ${\mathrm{L}-\mathrm{T}_{4}}_{4} 50 \mu \mathrm{g} /$ dia. Acompanhamento: $\mathrm{TSH}_{\mathrm{e}} \mathrm{T}_{4}$ estavam normais aos $51 \mathrm{dv}(\mathrm{C} 1)$ e aos $36 \mathrm{dv}$ (C2) sendo então gradativamente reduzida a dose da medicação e suspensa aos 16 meses (C1) e aos dez meses (C2). As pacientes foram acompanhadas até 22 meses (C1) e 16 meses (C2) com testes de função tireoidiana normais. O crescimento e o desenvolvimento neuropsicomotor (DNPM), avaliados pelo teste CAT/CLAMS, eram normais. Conclusão: As avaliações da função tireoidiana e do DNPM são necessários quando a AMD é utilizada na gestação. O tratamento do HCT deve ser instituído tão logo o diagnóstico seja realizado. (Arq Bras Endocrinol Metab 2008;52/1:126-130)

Descritores: Hipotireoidismo congênito transitório; Amiodarona; Hipotireoidismo congênito; Gestação; Desenvolvimento neuropsicomotor

\section{ABSTRAT}

Transient Neonatal Hypothyroidism Due to Amiodarone Administration During Pregnancy - Two Cases Report and Review of Literature.

Introduction: Amiodarone (AMD) is an antiarrhythmic agent which contains $37 \%$ of iodine. It can reach the fetus by transplacental passage and induce transient congenital hypothyroidism $(\mathrm{TCH})$. We report two cases of $\mathrm{TCH}$ caused by gestational exposure to AMD, detected by the Newborn Screening Program for Congenital Hypothyroidism of the State of Paraná - Brazil. Clinical case 1 (C1): Neonatal TSH value was 78,2 $\mathrm{mU} / \mathrm{L}$ (normal $<15 \mathrm{mU} / \mathrm{L}$ ). AMD had been given to the mother during pregnancy to treat maternal arrhythmia. The screening results were confirmed by serum thyroid function tests. Levothyroxin $\left(\mathrm{L}-\mathrm{T}_{4}\right)(50 \mu \mathrm{g} /$ day $)$ was started on the first visit, on the 14 th day of life (dl). Clinical case 2 (C2): Neonatal TSH value was 134,0 mU/L. AMD had been given to the mother in the third trimester of pregnancy to treat maternal arrhythmia. The screening results were confirmed by serum thyroid function tests: $L-T_{4}(50 \mu \mathrm{g} /$ day $)$ was started on the first visit, with $13 \mathrm{dl}$. Followup: TSH and $\mathrm{T}_{4}$ normalized on $51 \mathrm{dl}(\mathrm{C} 1)$ and $36 \mathrm{dl}$ (C2); L-T 4 could be diminished gradually and stopped within 16 months (C1) and 10 months (C2). They were followed-up until 22 months (C1) and 16 months (C2) with normal thyroid function tests. Their growth and mental development, evaluated by the 
Cognitive Adaptive Test/Clinical Linguistic \& Auditory Milestone Scale (CAT/ CLAMS test), were normal. Conclusion: Evaluation of thyroid function and mental development should be performed if AMD is used during pregnancy. Treatment of TCH must be started as soon as the diagnosis is made. (Arq Bras Endocrinol Metab 2008;52/1:126-130)

Keywords: Transient congenital hypothyroidism; Amiodarone; Congenital Hypothyroidism; Pregnancy; Mental development

\section{INTRODUÇÃO}

A AMIODARONA (AMD) é uma droga muito utilizada como um agente antiarrítmico que contém 37,5\% de iodo. É um derivado diiodinado do benzofurano e tem estrutura molecular parecida com a dos hormônios tireoidianos $\mathrm{T}_{3}$ e $\mathrm{T}_{4}$ (1). A AMD pode causar tireotoxicose, geralmente em áreas deficientes de iodo, e hipotireoidismo em áreas suficientes de iodo (2). É utilizada durante a gestação para o tratamento de taquiarritmias maternas ou fetais; atravessa a barreira placentária e distribue-se em vários tecidos, principalmente tecido adiposo, fígado, pulmão e cérebro (3). Sua meia-vida é longa, aproximadamente cem dias, em virtude de seu depósito no tecido adiposo, e por isso pode alcançar o feto após longo período de uso ou após ter sido suspensa (1). Como o feto adquire a capacidade de escapar do efeito Wolff-Chaikoff, somente no final da gestação uma oferta aumentada de iodo pode causar hipotireoidismo fetal/neonatal, bócio (3), retardo de crescimento intra-uterino e bradicardia fetal (4). O hipotireoidismo fetal causa alterações do DNPM, pois os hormônios tireoidianos são essenciais para o desenvolvimento cerebral (5). Foram também descritos casos de hipotireoidismo fetal decorrentes da infusão de AMD diretamente na circulação fetal (6) para o tratamento de taquiarrítmias fetais. Anormalidades neurológicas, decorrentes de um efeito neurotóxico da própria AMD, independentes de hipotireoidismo, foram descritas em poucas crianças expostas à AMD intra-útero (7).

Embora o hipotireoidismo causado pela AMD seja transitório, o tratamento com ${\mathrm{L}-\mathrm{T}_{4}}_{4}$ é necessário. $\mathrm{O}$ aleitamento materno, quando a mãe faz tratamento com AMD, está associado com uma ingestão substancial da droga pela criança, mas os poucos lactentes seguidos não apresentaram alterações da função tireoidiana (3).
Relatamos dois casos de HCT que foram encaminhados para a Unidade de Endocrionologia Pediátrica do Departamento de Pediatria do Hospital das Clínicas da Universidade Federal do Paraná (UEP-HC-UFPR) por apresentarem triagem neonatal positiva para $\mathrm{HC}$ e, durante a anamnese materna, foi constatado o uso de AMD durante a gestação. O DNPM das duas crianças foi avaliado pelo teste CAT/CLAMS (8).

O Programa de Triagem Neonatal para HC, no Estado do Paraná, foi implantado em 1990 pelo Centro de Pesquisas da Fundação Ecumênica de Proteção ao Excepcional (FEPE), em Curitiba. A amostra de sangue é obtida por punção de calcanhar em papel-filtro (Schleicher \& Schuell $2992^{\circledR}$ ), pela equipe de enfermagem nos hospitais, no momento da alta hospitalar. Naqueles casos em que a alta hospitalar ocorre antes de 48 horas de vida, o exame é repetido entre o quinto e o sétimo dia de vida nas Unidades de Saúde. As amostras são remetidas, via correio, ao Centro de Pesquisas da FEPE. As dosagens de TSH são realizadas em uniplicata, por kit DELFIA ${ }^{\circledR}$ hTSH neonatal, por ensaio imunofluorimétrico. No início do programa, o valor de corte considerado era de 20 $\mathrm{mU} / \mathrm{L}$, passando posteriormente para 15 e $10 \mathrm{mU} / \mathrm{L}$. Atualmente, repete-se a dosagem nas amostras com valores de TSH acima de $10 \mathrm{mU} / \mathrm{L}$ (ou acima de 15 $\mathrm{mU} / \mathrm{L}$, quando a coleta foi feita antes de 48 horas de vida). Se o valor for maior do que $30 \mathrm{mU} / \mathrm{L}$, a criança é imediatamente convocada para consulta médica. Se for menor do que $30 \mathrm{~m} \mathrm{U} / \mathrm{L}$, é solicitada nova amostra. Se, na reconvocação, o TSH for maior do que $10 \mathrm{mU} / \mathrm{L}$, a criança também é encaminhada para consulta. A convocação dos pacientes para consulta é realizada pela equipe de Serviço Social da FEPE.

\section{CASO CLÍNICO 1}

STM, sexo feminino, branca, apresentou triagem neonatal positiva para HC. Peso de nascimento: $3.450 \mathrm{~g}$, 
comprimento: $47 \mathrm{~cm}$, idade gestacional de 39 semanas. O TSH neonatal, com 48 horas de vida era 78,2 mU/L (normal < $15 \mathrm{mU} / \mathrm{L})$. A AMD, $200 \mathrm{mg} / \mathrm{dia}$, foi administrada à mãe durante toda a gestação por apresentar arritmia cardíaca. A função tireoidiana materna era normal: TSH, 4,5 mU/L, (0,4 - 5,0 mU/L); $\mathrm{T}_{4}$ total $\left(\mathrm{TT}_{4}\right), 12,4 \mu \mathrm{g} / \mathrm{dL}(6$ a $16 \mu \mathrm{g} / \mathrm{dL}$ ). Com $14 \mathrm{dias}$ de vida, a paciente foi referida para consulta na UEPHC-UFPR. Apresentava freqüência cardíaca (FC) de $150 \mathrm{bpm}$, peso, comprimento e perímetro cefálico normais. O resultado da triagem neonatal foi confirmado pelas dosagens séricas: TSH: $94 \mathrm{mU} / \mathrm{L} ; \mathrm{T}_{4} \mathrm{~L}$ : 0,2 ng/

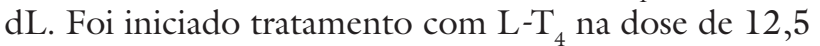
$\mu \mathrm{g} / \mathrm{kg} / \mathrm{dia}$ ( $50 \mu \mathrm{g} / \mathrm{dia})$. Aos 51 dias de vida, os exames de função tireoidiana eram: TSH, 5,5 mU/L, TT $16,7 \mu \mathrm{g} / \mathrm{dL}$; a dose de $\mathrm{L}_{-} \mathrm{T}_{4}$ foi diminuída para 5,8 $\mu \mathrm{g}$ / $\mathrm{kg} /$ dia $(25 \mu \mathrm{g} /$ dia $)$. O aleitamento materno foi exclusivo até 5 meses, sendo suspenso com 1 ano de vida. Aos 16 meses de idade, o tratamento foi suspenso. A paciente recebeu alta com 22 meses de idade com testes de função tireoidiana normais (TSH: 1,53 mU/L; T $\mathrm{L}$ : $1,45 \mathrm{ng} / \mathrm{dL}$ ). O crescimento (escores $\mathrm{Z}$ de peso: 1,62 e de estatura: 0,1 ) e o desenvolvimento mental (CAT/ CLAMS: 87,5; valor de referência: > 80) eram adequados para a idade da criança (Tabela 1 ).

\section{CASO CLÍNICO 2}

VASLO, sexo feminino, branca, apresentou triagem neonatal positiva para HC. Peso de nascimento: $3.090 \mathrm{~g}$, comprimento: $48 \mathrm{~cm}, 38$ 1/2 semanas de idade gestacional. TSH neonatal, 48 horas de vida, 134,0 mU/L. A mãe recebeu $\mathrm{AMD}, 100 \mathrm{mg} /$ dia, durante o último trimestre da gestação em virtude de taquicardia supraventricular. A função tireoidiana materna era normal: TSH, 0,59 $\mathrm{mU} / \mathrm{L} ; \mathrm{TT}_{4}, 12,8 \mu \mathrm{g} / \mathrm{dL}$. Aos 13 dias de vida a paciente foi encaminhada à UEP. Apresentava peso, comprimento e perímetro cefálico normais. A FC na primeira consulta era de $144 \mathrm{bpm}$. As dosagens séricas iniciais foram: TSH, $75 \mathrm{mU} / \mathrm{L} ; \mathrm{T}_{4} \mathrm{~L}, 0,2 \mathrm{ng} / \mathrm{dL}$; e $\mathrm{TT}_{4}, 1,0 \mu \mathrm{g} / \mathrm{dL}$. Foi iniciada $\mathrm{L}-\mathrm{T}_{4}, 11,3 \mu \mathrm{g} / \mathrm{kg} / \mathrm{dia}$ (50 $\mathrm{gg} / \mathrm{dia})$. Aos 36 dias de vida a função tireoidiana era: TSH, 0,1 mU/L, e TT $, 16,1 \mu \mathrm{g} / \mathrm{dL}$, e a dose de $\mathrm{L}-\mathrm{T}_{4}$ foi diminuída para $4,4 \mu \mathrm{g} / \mathrm{kg} / \mathrm{dia}(25 \mu \mathrm{g} / \mathrm{dia})$. Aos 10 meses de idade o tratamento foi suspenso. Aleitamento materno foi realizado até 10 meses de vida. Com 16 meses de idade, apresentava função tireoidiana normal (TSH, 2,4 mU/L; T $4,1,4$ ng/dL). O crescimento (escores $\mathrm{Z}$ de peso e estatura: $-0,3$ ) e o desenvolvimento mental (CAT/CLAMS: 100,5) eram normais (Tabela 1 ).

Tabela 1. Dados clínicos e laboratoriais dos casos clínicos 1 (C1) e 2 (C2) segundo a idade.

\begin{tabular}{|c|c|c|c|c|}
\hline & Idade & $\mathrm{Cl}$ & Idade & $\mathrm{C} 2$ \\
\hline TSH triagem (mU/L) & $2 d v$ & 78,2 & $2 d v$ & 134,0 \\
\hline TSH inicial (mU/L) & $14 d v$ & 94,0 & $13 d v$ & 75,0 \\
\hline $\mathrm{T}_{4} \mathrm{~L}$ inicial $(\mathrm{ng} / \mathrm{dL})$ & $14 d v$ & 0,2 & $13 d v$ & 0,2 \\
\hline Dose L-T $4(\mu \mathrm{g} / \mathrm{dia})$ & $14 d v$ & 50 & $13 d v$ & 50 \\
\hline TSH seguimento & $51 d v$ & 5,5 & $36 d v$ & 0,1 \\
\hline$\Pi_{4}$ seguimento $(\mu \mathrm{g} / \mathrm{dL})$ & $51 \mathrm{dv}$ & 16,7 & $36 \mathrm{dv}$ & 16,1 \\
\hline Dose L-T $(\mu \mathrm{g} / \mathrm{dia})$ & $51 d v$ & 25 & $36 d v$ & 25 \\
\hline TSH alta (sem medicação) & $22 \mathrm{~m}$ & 1,5 & $16 \mathrm{~m}$ & 2,4 \\
\hline $\mathrm{T}_{4} \mathrm{~L}$ alta (sem medicação) & $22 \mathrm{~m}$ & 1,4 & $16 \mathrm{~m}$ & 1,4 \\
\hline Escore Z do peso (alta) & $22 \mathrm{~m}$ & 1,6 & $16 \mathrm{~m}$ & $-0,3$ \\
\hline Escore Z da estatura (alta) & $22 \mathrm{~m}$ & 0,1 & $16 \mathrm{~m}$ & $-0,3$ \\
\hline Teste CAT/CLAMS & $14 \mathrm{~m}$ & 87,5 & $12 \mathrm{~m}$ & 100,5 \\
\hline
\end{tabular}

TSH e T L alta, ou seja, na ocasião em que se encerrou o seguimento. Teste CAT/CLAMS: avaliação do DNPM até os 3 anos de idade (valor normal de referência > 80); $d v$ = dias de vida; $m=$ meses. 


\section{DISCUSSÃO}

A AMD tem sido usada na gestação para os casos de taquiarritmias materno/fetais resistentes a outras drogas ou nos casos de risco de vida (4-9). No estudo sobre os efeitos adversos da AMD em 34 grávidas, Widerhorn e cols. (10) relataram três casos (9\%) de hipotireoidismo neonatal. Bartalena e cols. (3) revisaram 64 casos de recém-nascidos de mães que usaram AMD na gestação, encontrando 11 crianças (17\%) com HCT, das quais duas apresentavam bócio. A avaliação do DNPM foi normal em duas crianças e mostrou algum grau de comprometimento intelectual em três; as demais não foram avaliadas.

Magee e cols. descreveram nistagmo associado com movimentos anormais da cabeça em um de seis recémnascidos expostos a AMD no primeiro trimestre da gestação (4). Porém esses autores referem que a relação entre o uso da AMD e essas alterações permanece especulativa, pois podem estar presentes em $1 \%$ a $3 \%$ de todos os recém-nascidos. Na reavaliação de dez crianças cujas mães receberam AMD, Magge e cols. encontraram atraso global do DNPM em uma das crianças e outra que apresentava a "síndrome de inabilidade de aprendizado não-verbal” (7). O mesmo grupo relatou o caso de uma criança exposta a AMD a partir da $20^{a}$ semana de gestação que apresentou atraso no desenvolvimento, hipotonia, hipertelorismo e micrognatia na ausência de hipotireoidismo (4). Segundo esses autores, essas alterações seriam decorrentes de ação tóxica/ teratogênica da própria AMD (7).

A disfunção tireoidiana causada pela AMD não é dose-dependente. A AMD age inibindo a atividade da enzima 5'-deiodinase tipo $\mathrm{I}$, aumentando o $\mathrm{TT}_{4}$ e o $\mathrm{T}_{3}$ reverso e diminuindo a concentração sérica do $\mathrm{T}_{3}$. Age também sobre a atividade da enzima 5'-deiodinase tipo II interferindo com a interação da triiodotironina com seu receptor nuclear na hipófise, causando aumento do TSH. Inibe a entrada do hormônio tireoidiano nas células, diminuindo a produção periférica de $\mathrm{T}_{3}$, é citotóxica para a tireóide e interage com os receptores do hormônio tireoidiano, diminuindo a resposta dos genes responsivos ao hormônio tireoidiano, causando uma condição tecidual de hipotireoidismo (1). Indivíduos com anormalidades da hormoniogênese tireoidiana e aqueles com anticorpos antitireoidianos são mais susceptíveis a apresentar alterações tireoidianas com o uso de AMD (1).
A tireóide fetal desenvolve-se em vários estágios. Até a $11^{\underline{a}}$ e a $12^{\underline{a}}$ semanas de gestação, a secreção adequada de hormônio tireoidiano pela mãe é necessária para o feto. Conseqüentemente durante o primeiro trimestre de gestação o fornecimento de hormônio tireoidiano materno é essencial. Durante o segundo e o terceiro trimestre de gestação, a fonte de iodo é tanto de origem materna como fetal. A partir da $11^{\underline{a}}$ e da $12^{\underline{a}}$ semanas de gestação, a tireóide fetal inicia a captação de iodo, mas a glândula tireoidiana ainda não é capaz de limitar a captação e o transporte de iodo. Um excesso de iodo intracelular pode resultar inibição da síntese de hormônios tireoidianos - efeito Wolff-Chaikoff. Somente nas últimas quatro semanas da gestação a tireóide fetal é capaz de escapar do efeito Wolff-Chaikoff. A tireóide fetal se apresenta de 20 a 50 vezes mais ávida por iodo do que a tireóide materna. Tão pouco como $12 \mathrm{mg}$ de iodo pode produzir bócio neonatal. De Wolf e cols. descrevem um caso de HC transitório e bócio associado ao uso de AMD durante a gestação (11).

Vanbesien e cols. descreveram dois casos da infusão de AMD diretamente na circulação venosa fetal para o tratamento de arritmia cardíaca fetal. Houve desenvolvimento de hipotireoidismo intra-útero, sendo que o tratamento com $\mathrm{L}_{-} \mathrm{T}_{4}$ foi iniciado no período pré-natal por via amniótica (6). Essas crianças apresentaram DNPM normal.

$\mathrm{O}$ risco de desenvolver hipotireoidismo neonatal associado ao uso de AMD foi estimado por Magee e cols. (4) como sendo de 9\% (uma criança em 11 expostas à $\mathrm{AMD}$ na gestação). Esse risco não estava relacionado com a dose da $\mathrm{AMD}$, duração da terapia e confirmação da passagem da AMD via transplacentária (4). O risco é similar ao dos adultos tratados com AMD que é de $5 \%$ a $8 \%$.

Nas pacientes deste estudo não houve relação de dose ou duração da terapia com AMD com o desenvolvimento de HCT. No primeiro caso, a mãe utilizava AMD desde o início da gestação, e no segundo caso, a mãe recebeu AMD no último trimestre da gestação. Provavelmente as mães estavam eutireoidianas durante a gestação, pois apresentavam valores normais dos hormônios tireoidianos logo após o parto.

Segundo Matsumura e cols., a AMD pode ser utilizada durante a gestação nas mulheres com taquicardia resistente a outros tratamentos, desde que a função tireoidiana do neonato seja avaliada; em nove casos estudados por esses autores, nos quais as mães utilizaram 
AMD na dose de $200 \mathrm{mg} /$ dia, foi observado apenas um caso de HCT (12).

Foram descritos casos de pequenos para a idade gestacional (4) decorrentes de uso de AMD na gestação. Como o baixo peso de nascimento também está relacionado com doenças cardíacas e com o uso de outras drogas, tais como os betabloqueadores, a presença destes fatores torna improvável implicar a AMD como sendo a única etiologia para o baixo peso ao nascimento.

As duas pacientes deste estudo apresentavam exame clínico normal ao nascimento, peso e talhe de nascimento adequados para a idade gestacional e foram diagnosticadas portadoras de HC pelo exame da triagem neonatal, confirmado com as dosagens de TSH e de $\mathrm{T}_{4} \mathrm{~L}$. Em ambas, os níveis de TSH normalizaram com 36 e 51 dias após a instituição de $\mathrm{L}_{-} \mathrm{T}_{4}$. Foram descritos recém-nascidos com bradicardia decorrente do uso de AMD pela mãe (4), o que não foi observado nas pacientes deste estudo.

Quando o aleitamento materno for permitido, a avaliação da função tireoidiana é necessária em virtude de contínua exposição da criança a AMD. Há elevação da concentração de AMD e de seu metabólito ativo a desetilamiodarona (DEA) no leite materno (13), que pode ser explicado pela alta solubilidade lipídica desse metabólito. Foi estimado que o lactente pudesse ingerir $1,5 \mathrm{mg}$ de AMD $/ \mathrm{kg} /$ dia pela lactação (3). A expressão de NIS foi encontrada em glândulas mamárias em lactação e o iodo é ativamente transportando pelo tecido mamário e secretado pelo leite. Estudos em ratazanas tratadas com altas doses de iodo mostraram que os filhotes em amamentação apresentavam hipotireoidismo e bócio (14).

Concluindo, a alteração da função tireoidiana parece ser independente da dose e da duração da terapia com AMD. A AMD é uma droga eficaz no tratamento de taquiarritmias resistentes, porém, parece ser prudente não associar terapia concomitante com betabloqueadores. Uma avaliação apropriada da função tireoidiana dos recém-nascidos expostos à AMD é imperativa. O tratamento do HCT, tão logo o diagnóstico seja feito, mesmo por curto período, é crucial para se evitar alterações no crescimento e no desenvolvimento mental do recém-nascido. A amamentação pode ser permitida, porém uma avaliação periódica da função tireoidiana deverá ser realizada.

As duas pacientes deste estudo apresentaram HCT, foram tratadas adequadamente e apresentaram cresci- mento e DNPM normais. Definir se a neurotoxicidade da AMD per se pode estar associada com atraso no DNPM ainda precisa ser estabelecido por meio do seguimento de outras crianças expostas a essa droga.

\section{REFERÊNCIAS}

1. Basaria S, Cooper DS. Amiodarone and the thyroid. Am J Med. 2005;118:706-14.

2. Grosso S, Berardi R, Cioni M, Morgese G. Transient neonatal hypothyroidism after gestational exposure to amiodarone: a follow-up of two cases. J Endocrinol Invest. 1998;21(10): 699-702.

3. Bartalena L, Bogazzi F, Braverman LE, Martino E. Effects of amiodarone administration during pregnancy on neonatal thyroid function and subsequent neurodevelopment. J Endocrinol Invest. 2001;24/2:116-30.

4. Magee LA, Downar E, Sermer M, Boulton BC, Allen LC, Koren G. Pregnancy outcome after gestational exposure to amiodarone in Canada. Am J Obstet Gynecol. 1995; 172/4:1307-11.

5. Rovet JF, Ehrlich RM, Sorbara DL. Neurodevelopment in infants and preschool children with congenital hypothyroidism: etiological and treatment factors affecting outcome. J Pediatr Psychol. 1992;17:187-213.

6. Vanbesien J, Casteels A, Bougatef A, De Catte L, Foulon W, De Bock S, et al. Transient fetal hypothyroidism due to direct fetal administration of amiodarone for drug resistant fetal tachycardia. Am J Perinatol. 2001;18(2):113-6.

7. Magge LA, Nulman I, Rovet JF, Koren G. Neurodevelopment after in utero amiodarone exposure. Neurotoxicol Teratol 1999;21:261-5.

8. Wechsler D. WISC-III - Escala de inteligência Wechsler para crianças: Manual, 3.ed. Figueiredo VLM. Adaptação e padronização de uma amostra brasileira. 1.ed. São Paulo, 2002.

9. Foster CJ. Amiodarone in pregnancy. Am J Cardiol. 1994; 74:307.

10. Widerhorn J, Bhandari AK, Bughi S, Rahimtoola SH, Elkayam $U$. Fetal and neonatal adverse effects profile of amiodarone treatment during pregnancy. Am Heart J. 1991;122(4 pt1):1162-6.

11. De Wolf D, De Schepper J, Verhaaren H, Deneyer M, Smitz J, Sacre-Smits L. Congenital hypothyroid goiter and amiodarone. Acta Paediatr Scand. 1988;77/4:616-8.

12. Matsumura LK, Born D, Kunii IS, Franco DB, Maciel RM. Outcome of thyroid function in newborns from mothers treated with amiodarone. Thyroid. 1992;2/4:279-81.

13. McKenna WJ, Harris L, Rowland E, Whitelaw A, Storey G, Holt D. Amiodarone therapy during pregnancy. Am J Cardiol. 1983;51:1231-3.

14. Markou K, Georgopoulos N, Kyriazopoulou V, Vagenakis AG. lodine-Induced hypothyroidism. Thyroid. 2001;11/5:501-10.

Endereço para xorrespondência:

Luiz de Lacerda Filho

Rua Padre Camargo, 250

80060-240 Curitiba, PR

E-mail: Ilacerda@ufpr.br 